\title{
Amours privées, amours publiques, amours publiées ; l'inscription de divers cercles publics dans quelques avatars des canzonieri pétrarquistes des années 1570-1580
}

\author{
CHARLOTTE TRIOU
}

Université Lumière-Lyon 2

Cet article propose une lecture des indices qui, dans des recueils imprimés, formulent une adresse à un public restreint et se réfèrent à une circulation préalable des pièces poétiques. Il s'agit d'analyser la tension, du point de vue de la réception par des publics successifs, entre le paradigme de lecture générique du canzoniere et l'inscription dans un contexte éthique et social particulier. Sont ainsi comparés des itinéraires de publication très différents: d'une part, des processus ancrés dans le contexte de la cour royale (Jamyn et Ronsard), et d'autre part, des processus naissant du deuil d'une épouse (Christofle du Pré et Pierre de Brach). Ces recueils qui détournent le cadre énonciatif pétrarquiste et étendent les significations des motifs pétrarquistes permettent d'observer la concurrence entre les attentes herméneutiques liées au genre du canzoniere et celles liées à l'adresse à un lectorat de circonstance.

This article proposes an analysis of the clues given in printed collections by their address to a limited public and by the circulation of individual poems prior to publication. We will analyze, from the point of view of reception by successive audiences, the tension between the paradigm of generic reading of the canzoniere and a published work's inscription in a specific ethical and social context. Two very different avenues of publication are compared-involving, on the one hand, processes rooted in the context of the royal court (Jamyn and Ronsard), and on the other hand, those arising from a spouse's mourning (Christofle du Pré and Pierre de Brach). These collections, which readapt the discursive framework of Petrarchism and extend the significance of Petrarchan motifs, permit us to discern the competition between the interpretative expectations tied to the genre of the canzoniere and those tied to addressing an appropriate readership.

\footnotetext{
A partir de l'appropriation de l'œuvre de Pétrarque, du pétrarquisme italien et de la poésie amoureuse antique, les poètes français du milieu $\mathrm{du} \mathrm{XVI}^{\mathrm{e}}$ siècle se sont mis à composer des recueils qui chantent la femme aimée, selon un modèle qui va rester pratiqué jusqu'au début du XVII ${ }^{e}$ siècle. Une standardisation poétique et éditoriale des recueils amoureux s'opère en France, de sorte que les poètes qui s'emparent du genre jouent avec ses passages obligés et que le lectorat peut apprécier ces recueils en tant que public habitué à leurs conventions. Les critères sont bien sûr d'abord thématiques (l'amour
} 
pour une dame inaccessible) et formels (l'élection de la forme sonnet comme forme poétique privilégiée, quoique non exclusive). En outre, la composition des recueils est en perpétuelle tension entre la formation d'un tout cohérent et la discontinuité des poèmes. Cette tension empêche toute interprétation systématique des cohérences narratives ou logiques entre les poèmes mis en série et permet une hétérogénéité des adresses et des tonalités à l'intérieur d'un même recueil. C'est ce qu'étudie Cécile Alduy dans Politique des Amours ${ }^{1}$, pour la période 1544-1560, étude complétée par celle de Daniele Maira dans Typosine, la dixième muse ${ }^{2}$, pour la même période et sur les similarités de présentation qui apparaissent dans la fabrique éditoriale des livres, par exemple l'habitude de faire figurer des portraits de l'auteur et de la dame au début des livres, et de placer, à partir des Euvres de 1560 de Ronsard, les "Amours » comme sections inaugurales au sein d'une ouvre poétique plus vaste.

Dans les décennies 1570-1580, la première vague d'essor des canzonieri français est passée, et le lectorat des poètes français est familier de ces recueils. C'est au sein de la production poétique de cette période que quatre œuvres ont retenu notre attention, parce qu'elles proposent des détournements particuliers de la forme du canzoniere amoureux.

Ces quatre œuvres sont d'une part les poèmes d'Amadis Jamyn $(1575)^{3}$ et de Pierre de Ronsard (1578) ${ }^{4}$ sur les amours d'Eurymedon et de Callirée (c'està-dire du roi Charles IX et d'Anne d'Acquaviva), et d'autre part les poèmes de Christofle du Pré (1577) 5 et de Pierre de Brach (composition manuscrite, terminus a quo vers 1587 - terminus ad quem 1605$)^{6}$ écrits à la suite de la mort

1. Cécile Alduy, Politique des Amours (Genève : Droz, 2007).

2. Daniele Maira, Typosine, la dixième muse (Genève : Droz, 2007).

3. Les Euvres poetiques d'Amadis Jamyn, au Roy de France et de Pologne (Paris : Robert Estienne et Mamert Patisson, 1575). Édition moderne citée : Amadis Jamyn, Les Euvres poétiques, livres II, III et IV, éd. Samuel M. Carrington (Genève : Droz, 1978).

4. Les Euvres de Pierre de Ronsard Gentilhomme Vandomois (Paris : Gabriel Buon, 1578). Édition moderne citée : Pierre de Ronsard, Euvres complètes, vol. 17-2, éd. Paul Laumonier, révision I. Silver et R. Lebègue (Paris : Librairie Marcel Didier, 1959).

5. Les larmes funebres de Christofle du Pré Parizien Sieur de Passy (Paris : Robert Estienne et Mamert Patisson, 1577). Édition moderne citée : Christofle du Pré, Les Larmes funebres, éd. Pierre Martin (Genève : Droz, 2004).

6. Bibliothèque municipale de Bordeaux, ms. 1667 (voir Catalogue des manuscrits de la Bibliothèque municipale de Bordeaux, vol. 1, de la cote Ms 1 à la cote Ms 3171 : http://bibliotheque.bordeaux. 
de leur épouse. Ce sont des « avatars » de canzonieri, à la limite de l'inscription générique, mais dont les conditions d'intelligibilité tiennent en partie au code poétique des canzonieri. En effet, d'une part, chez Pierre de Ronsard et Amadis Jamyn, il s'agit d'une poésie amoureuse pour autrui, produite dans un contexte de cour, mais ces vers amoureux viennent pourtant s'intégrer ensuite aux recueils des œuvres des deux auteurs et prendre place dans la série des sections d'« Amours ». D’autre part, chez Christofle du Pré et Pierre de Brach, il s'agit d'une poésie amoureuse " de deuil », a priori inscrite dans la sphère intime, mais la publication de ces recueils de sonnets de deuil les fait entrer en écho avec la poésie pétrarquiste. En effet, si leurs canzonieri endeuillés sortent de la situation topique où l'amant exprime inlassablement son désir contrarié, la mort de la femme aimée est bien un événement central du Canzoniere de Pétrarque, puisque ce recueil est traditionnellement scindé en deux parties de part et d'autre de la mort de Laure, «In vita» et «In morte » ${ }^{7}$. La deuxième partie est ainsi consacrée aux plaintes et aux déchirements spirituels de l'amant après la mort de Laure. Cet événement de la mort de la dame a été repris en France par Ronsard dans ses sonnets «Sur la mort de Marie », mais il ne s'agit pas d'un deuil conjugal, motif jusqu'alors inédit en français; le motif existe cependant dans la poésie néo-latine, et plus précisément dans les Noenice de Salmon Macrin ${ }^{8}$.

L'enjeu de cet article est de mettre en lumière, au sein des recueils étudiés, la tension entre l'inscription d'un texte dans l'intertextualité des recueils amoureux (qui implique des attentes de lecture étant donné les habitudes des lecteurs face à un format de recueil relativement standardisé) et les traces de

fr/in/faces/documentViewer.xhtml?id=h::BordeauxPS_B330636101_CGM_01\&file=/in/rest/ annotationSVC/DownloadAttachment/attach_import_6bc33591-4d2b-4a70-8be5-4a6f44028c35?fileN ame=\%22B330636101_CGM_01.pdf\%22, 679). Éditions modernes citées : Euvres poétiques de Pierre de Brach, vol. 1, éd. Reinhold Dezeimeris (Paris : Aubry, 1861-1862) ; Pierre de Brach, Les Amours d’Aymée, éd. Jasmine Dawkins (Genève : Droz, 1978).

7. Pierre Blanc considère ainsi que Pétrarque est le premier à mettre en scène une crise spirituelle par la confrontation à la mort de la dame aimée, dans l'introduction de sa traduction : Pétrarque, Canzoniere - Le Chansonnier, éd. Pierre Blanc (Paris : Classiques Garnier, 1988), 32-34.

8. Noniarum libri tres, de Gelonide Borsala uxore charissima quce annos XXXX, menses II, dies XV nata, obiit XIII junii (Paris : Michel de Vascosan, 1550). Voir Perrine Galand-Hallyn, « Le "jour en trop" de Jean Salmon Macrin (l'ode liminaire des Nonice de 1550 : grandeur et plasticité) », in Devis d'amitié. Mélanges en l'honneur de Nicole Cazauran, éd. Jean Lecointe, Catherine Magnien, Isabelle Pantin et Marie-Claire Thomine (Paris : Honoré Champion, 2002), 525-547. 
circulation des textes pour des publics restreints, qui peuvent entrer en conflit avec la lecture qu'un public plus large peut faire des recueils. Nous nous inspirons ici de Roger Chartier, lorsqu'il étudie la façon dont la stabilité ou l'instabilité du texte, dans sa lettre ou dans sa forme imprimée, crée de nouvelles « lisibilités » et donc constitue de nouveaux publics (et réciproquement) ${ }^{9}$. Ce n'est pourtant pas l'instabilité des modifications textuelles ou de la présentation éditoriale qui nous intéresse ici, mais celle qui provient du conflit entre deux systèmes d'intelligibilité : celui de l'événement social et celui du genre littéraire. Ce conflit permet de décliner plusieurs types de tension entre des publics : du point de vue de l'extension, une tension entre un public restreint et un public large ; du point de vue temporel, une tension entre un public immédiat et un public à venir ; du point de vue herméneutique, une tension entre un public de connivence relationnelle et un public de connivence culturelle.

En somme, il s'agit de réfléchir à la tension entre une herméneutique fondée sur l'adresse de circonstance et une herméneutique fondée sur le code générique. Il faut préciser que cette alternative problématique n'est pas propre aux « cas-limites » étudiés ici. En effet, comme le montre Cécile Alduy ${ }^{10}$, la superposition entre destinataire réel et destinataire fictif est constitutive de la poétique des canzonieri, qui jouent sur la correspondance ambiguë entre le personnage de la dame et le/la/les destinataire(s) de l'ouvrage. Mais dans ces quatre œuvres de Ronsard, Jamyn, Du Pré et Brach, l'inscription dans un ou plusieurs cercles publics ne relève pas seulement de la définition d'un ou d'une destinataire de l'œuvre, mais d'une véritable participation du public au processus de publication et à l'émergence des significations de l'œuvre.

Nous commencerons par présenter les processus de publication des deux sections d'œuvres de Ronsard et Jamyn sur les amours d'Eurymédon et Callirée, puis des deux recueils « funèbres » de Brach et Du Pré. L'horizon de l'analyse sera de mettre en lumière la façon dont ces processus s'inscrivent dans des temporalités historiques (événement/durée, péremption/éternisation...). Ensuite, nous reviendrons à l'analyse littéraire afin de la mettre à l'épreuve, en cherchant à fonder les conditions de signification des pièces poétiques sur la prise en compte des modalités de publication. Nous prendrons pour exemples

9. Roger Chartier, "Communautés de lecteurs ", in Culture écrite et société : l'ordre des livres (XIVEXVIII siècle) (Paris : Albin Michel, 1996), 141-147.

10. Cécile Alduy, "Lecteurs réels et lectrices alléguées : lire les recueils d'Amours au XVI ${ }^{e}$ siècle ", $L a$ Lecture littéraire. Revue de recherche sur la lecture des textes littéraires 7 (2003) : 119. 
les adaptations de la dialectique verticale qui s'instaure dans la relation entre l'amant et la dame.

\section{Récits de publication : extensions et restrictions des publics, temporalités des publications}

Nous entendons le terme de "publication ", suivant Christian Jouhaud et Alain Viala ${ }^{11}$, au sens large d'acte de partage et de divulgation d'un texte, afin d'élaborer des " récits de publication " qui reconstituent des " chaînes de publication » par un recensement des étapes successives par lesquelles un texte peut se trouver " publié » selon différentes modalités. Il s'agit de sortir d'un schéma binaire qui met en relation l'auteur et un vaste lectorat indéterminé, pour redonner à chaque interaction autour du texte sa valeur d'acte de publication. En ce sens, l'alternative " amours privées, amours publiques » se résout en « amours publiées ", quel que soit le caractère plus ou moins intime ou officiel des relations qui unissent les acteurs impliqués.

La diversité des modalités de publication invite à considérer ces quatre ensembles de poèmes dans leur instabilité. En effet, il s'agit de poèmes, du moins en ce qui concerne Ronsard, Jamyn et Brach de manière certaine, qui circulent d'abord de manière éparse (c'est-à-dire non réunis en œuvres collectives) et sous la forme de premières versions manuscrites ou lues en performance orale avant qu’une édition imprimée en soit donnée. L'édition en recueil imprimé, qui nous parvient après plusieurs siècles, n'est qu'une des modalités qui rendent public un poème, et c'est celle qui fait entrer le poème dans les cadres herméneutiques liés à la lecture des recueils pétrarquistes. Mais une édition imprimée ne fixe pas non plus définitivement ce cadre herméneutique, et il importe de la considérer comme état provisoire de publication du texte, promis à devenir daté. Ainsi, ce que montreront également les « récits de publication » des poèmes, ce sont les tensions entre plusieurs temporalités du processus d'écriture et de publication : que signifie une publication imprimée différée par rapport à une lecture orale des poèmes ? que signifie au contraire une publication imprimée quasi immédiatement après l'événement auquel se réfère un texte ? que signifie une publication différée, voire à jamais retenue ? Les réponses à ces questions seront

11. Voir De la Publication entre Renaissance et Lumières, éd. Christian Jouhaud et Alain Viala (Paris : Fayard, 2002), 14-18. 
bien différentes d'une œuvre à une autre, mais les deux catégories d'œuvres analysées ici, œuvres qui célèbrent plaisamment un événement de cour et œuvres qui «accomplissent» un deuil, la tension entre la circonstance et la permanence, entre l'événement advenu et la durée que lui donne l'écriture, est particulièrement significative.

\section{Amours de cour}

Amadis Jamyn, dans les Euvres poetiques de 1575, et Pierre de Ronsard, dans les Euvres de 1578, ont fait chacun paraitre une section consacrée aux amours de Charles IX (sous le nom d'Eurymedon, du grec : « celui qui gouverne au loin / de loin ») et de Madame d'Acquaviva (sous le nom de Callirée, du grec également : «la belle eau qui coule » $)^{12}$. Ces deux poètes ont ainsi pris pour sujet les amours du roi Charles IX, réputé pour avoir été d’abord passionné de chasse et peu porté sur la galanterie. À l'origine de ces pièces amoureuses intervient probablement une commande pour le roi Charles IX (mais sans certitude sur l'identité du commanditaire). Cependant, la publication imprimée, postérieure de quelques années, éloigne la présence symbolique des destinataires premiers, qui deviennent personnages. Cet éloignement est d'autant plus évident que Charles IX décède en 1574 et qu'il est donc mort lorsque Jamyn et Ronsard publient leurs recueils. Jamyn dédie ainsi ses œuvres « Au Roy de France et de Pologne », à savoir à Henri III, qui a succédé à son frère. Difficile de savoir si ce délai observé avant publication est dû à un impératif de convenance courtisane ou à d'autres déterminations éditoriales.

L'enjeu du classement d'une section d'œuvres poétiques sous le titre «Amours » est important et c'est même une caractéristique du genre que d'être « encadré » par des seuils qui suggèrent une plus grande unité architecturale que n’en a en réalité le recueil, une " unité de façade », selon Cécile Alduy ${ }^{13}$, qui annonce une plus grande cohérence que ce que les poèmes mis en série produisent effectivement au sein des recueils. L'enjeu de l'intitulation est donc important : intituler un recueil « Amours » revient à l'inscrire dans une

12. Voir, pour Jamyn, Theodosia Graur, Un Disciple de Ronsard, Amadis Jamyn 1540-1593 : sa vie, son cuvre, son temps (Paris : Honoré Champion, 1929), 106-114 et, pour Ronsard, Marcel Raymond, «Sur les Amours d'Eurymedon et de Calliree », in De Jean Lemaire de Belges à Jean Giraudoux, Mélanges d'histoire et de critique littéraire offerts à Pierre Jourda (Paris : Nizet, 1970), 59-74.

13. Alduy, Politique des Amours, 313-354 et 387. 
certaine pratique partagée du recueil amoureux et crée un horizon d'attente pour le public.

Or, le poète qui souhaite recueillir des textes écrits sur commande dans ses œuvres se trouve devant une alternative : une première option est que le poète s'approprie cet amour et fasse du je lyrique une voix lyrique qui lui soit propre (ce quavait fait Ronsard par exemple pour ses sonnets « Sur la mort de Marie » composés à l'occasion de la mort de Marie de Clèves, pleurée par le futur Henri III, avant que le poète ne s'approprie cette " Marie » en publiant ses pièces en son nom pour le deuil de Marie de Bourgueil ${ }^{14}$ ); ou au contraire, une seconde option, qui s'applique à la section consacrée à Eurymedon et Callirée chez Ronsard comme chez Jamyn, est de rendre visible la composition «pour autrui ». L'évolution du titre atteste de ce choix. En effet, chez Jamyn, l'édition des œuvres recueillies en 1575 présentait une certaine ambiguïté dans l'intitulation car la section portait simplement le titre « Calliree », avec la mention « Troisieme livre » sans différence avec les deux autres livres d' " Amours » du poète que sont " Oriane » et « Artémis »; mais le titre devient « Les Amours d'Eurymedon et de Calliree » dans les rééditions de 1579 et $1582^{15}$, ce qui rétablit le caractère d'emprunt de cette « histoire d'amour » dont traite le poète. Le nom de la dame n'est plus seul, ce qui empêche toute attribution de l'amour au poète. Chez Ronsard, les deux protagonistes Eurymedon et Callirée sont mentionnés dès 1578, ce qui leur donne un statut légèrement à part dans la mise en série avec les autres sections d'« Amours". On observe en outre une évolution du titre qui marque une réticence à classer les pièces pour Eurymedon et Callirée dans la série des autres " Amours ", car à partir de la réédition de $1584^{16}$, la section "Amours d'Eurymedon et de Callirée " devient "Vers d'Eurymedon et de Callirée $»^{17}$.

14. Jean Vignes analyse ce procédé dans son article « Jean-Antoine de Baif, poète du deuil conjugal par procuration ", in Aspects du lyrisme conjugal à la Renaissance, éd. Perrine Galand et John Nassichuk (Genève : Droz, 2011), 210.

15. Les Euvres poétiques d'Amadis Jamyn (Paris: Mamert Patisson, 1579) ; Les Euvres poétiques d'Amadis Jamyn (Paris : Robert Estienne, 1582).

16. Les CEuvres de Pierre de Ronsard gentilhomme vandomois (Paris : Gabriel Buon, 1584).

17. Il faut bien sûr garder à l'esprit que ces enjeux d'intitulation peuvent être aussi traités avec l'imprimeur libraire ou d'autres personnes qui dialoguent avec Ronsard et qui participent à l'élaboration de la forme imprimée du texte. 
Cette mise à distance et cet éloignement du premier public constitué par les destinataires des poèmes sont également sensibles dans le fait que Claude Binet, premier biographe de Ronsard, juge utile d'expliquer dans sa Vie de Ronsard comment ces pièces ont été " forgées sur le commandement des Grans ${ }^{18}$. Claude Binet évoque ces pièces poétiques dans les deuxième et troisième versions de cette Vie, qui sont publiées conjointement aux éditions posthumes des Euvres de Ronsard en 1587 et $1597^{19}$. Ces vers pour Eurymedon et Callirée sont ancrés dans une circonstance que les lecteurs qui n'étaient pas contemporains des événements ne peuvent comprendre sans une note d'érudition donnant la «clé » mondaine du texte. Claude Binet y pourvoit donc, mais de deux façons différentes dans les deux versions successives de sa Vie de Ronsard:

En $1587:[\ldots]$ personne n'ignore en faveur de qui il fit les Amours d'Eurimedon et de Callirée [...].

En $1597:[\ldots]$ on sçait assez en faveur de qui il fit les Amours de Callyrée, qui estoit une très belle dame de la Cour, de la noble maison d'Atry, surnommée Aqua viva : comme il l'exprime assez en ce Sonet qui commence, La belle eau vive [...]. ${ }^{20}$

La première version fait appel à une connivence, tandis que la seconde ne tient plus pour acquise la connaissance de l'identité de Callirée. Ces précisions données par Claude Binet révèlent l'évolution progressive du public des Euvres de Ronsard, dans la mesure où ce public s'élargit et devient de moins en moins compétent pour déchiffrer les clés du texte et apprécier ce dispositif de clés ${ }^{21}$. Ces ajouts contribuent en outre à historiciser ces vers en suggérant qu'ils ne peuvent être extraits d'un cadre de poésie de circonstance.

Ainsi, les deux modalités de publication qui s'opposent, dans le cas de ces vers courtisans, sont le partage mondain et l'intégration à un recueil d'œuvres. Dans

18. Les CEuvres de Pierre de Ronsard gentilhomme vandomois (Paris : Gabriel Buon, 1587), X, 129.

19. Claude Binet, La Vie de P. de Ronsard (Paris : Hachette, 1910). La première édition (1586) n'en faisait pas mention.

20. Variantes relevées par Paul Laumonier, dans Binet, La Vie de P. de Ronsard, 25.

21. Sur cette question des clés, voir Mathilde Bombart et Marc Escola, «Clés et usages de clés : pour servir à l'histoire et à la théorie d'une pratique de lecture », Littératures classiques 54 (2004) : 5-26. 
la lecture du recueil, cette opposition subsiste à l'état de tension herméneutique, entre une herméneutique de clés liée au souvenir de l'événement et une herméneutique générique liée au déplacement du code poétique pétrarquiste, sur lequel nous reviendrons.

$\mathrm{Si}$, dans le cas des recueils de vers courtisans, la poésie naît de la présence première du destinataire, dans le cas des vers "funèbres », c'est au poète de travailler à constituer et rassembler un public.

\section{Deuils conjugaux}

À partir d'un événement similaire, celui du décès de l'épouse, Pierre de Brach et Christofle du Pré ont deux attitudes différentes quant à la temporalité de la publication. En effet, à la mort de sa femme Anne Perrot (1587), Pierre de Brach travaille longuement son recueil et ne le fera pas imprimer, faisant durer une circulation manuscrite en cercle restreint, et construisant progressivement un ouvrage où ses " larmes » répondent à un " tombeau » collectif, dans une temporalité longue qu'il revendique lui-même comme un engagement éthique dans un deuil de longue durée. Au contraire, Christofle du Pré publie très rapidement son recueil de 75 sonnets, comme dans l'urgence d'élever le monument à sa femme défunte, Antoinette de Faucon (1577).

Entrons quelque peu dans le détail de ces deux processus de publication et voyons combien ils résonnent avec deux poétiques et deux éthiques de la poésie bien différentes.

Chez Pierre de Brach, les « Regrets et larmes funebres sur la mort d'Aymée ", font partie d'un manuscrit préparatoire à une édition ${ }^{22}$, qui intègre des poèmes amoureux déjà publiés en 1576 dans les Poemes ${ }^{23}$. Cette édition de 1576 est en bonne partie disloquée dans le manuscrit, et s'y ajoutent un deuxième livre d' " amours ", un troisième livre de "regrets et larmes funebres » et un quatrième livre recueillant des poèmes allographes pour un " tombeau » collectif à Aymée. Il est important de souligner que c'est la figure d'Aymée qui donne sa cohérence aux quatre livres prévus, d'abord sous les traits conventionnels de la «dame », puis en tant qu'épouse. L'ensemble était destiné à produire un monument dialogique complexe étant donné qu'un certain nombre de poèmes des « regrets et larmes funebres » étaient adressés à des personnes

22. Bibliothèque municipale de Bordeaux, ms. 1667.

23. Les poemes de Pierre de Brach Bourdelois (Bordeaux : Simon Millanges, 1576). 
ayant envoyé leur contribution à ce tombeau. Le manuscrit du poète est décrit par Jasmine Dawkins (éditrice des Amours d'Aymée) comme un manuscrit de travail $^{24}$, mais les pièces qui le composent ont, au moins partiellement, circulé. Le manuscrit est en réalité aussi un manuscrit à faire lire. En effet, si la dédicace de Pierre de Brach à Monsieur de Nesmond est écrite de la main d'un copiste, le titre, la formule finale et la signature sont de la main du poète ${ }^{25}$ : ce soin de signer en personne laisse à penser que ce manuscrit avait un statut hybride, $\grave{a}$ la fois manuscrit de travail (pièces ou dédicaces biffées, pièces découpées et déplacées...) et manuscrit communicable à un cercle restreint de personnes. Il s'agit donc d'une publication.

Cette circulation des poèmes prend place dans un système de don / contredon $^{26}$, comme le suggère Pierre de Brach à la fin de la dédicace à Monsieur de Nesmond :

[...] de sa mort je n'ay jusques icy tiré autre contentemant que celuy que j'ay maintenant de vous offrir ces larmes, lesquelles, de leurs espineuses plantes, ont faict naistre tant de belles et riches fleurs qui embaument son tombeau que je vous offre aussi ; ce sera en contre-eschange de celles de vostre jardin dont il vous a pleu me faire part que j'ayme, non tant pour leur rareté, pour leur beauté ou pour leur odeur que pour ce qu'en leurs metamorphoses quelque mort est représentee $[\ldots .]^{27}$

Le processus de don / contre-don entre Brach et Nesmond est complexe, car les deux hommes se gratifient de poèmes dans une ronde qui s'entretient longuement, dont il est possible de reconstituer certaines étapes :

24. Voir Jasmine Dawkins, "Les Manuscrits de Pierre de Brach ", Bibliothèque d'Humanisme et Renaissance 32.1 (1970) : 95-97, où est décrite la dislocation de l'édition imprimée dans le travail de recomposition du recueil.

25. Voir Brach, Les Amours d'Aymée, 269.

26. Dans la lignée du travail de Marcel Mauss sur les mécanismes de don / contre-don, Nathalie Zemon Davis analyse les pratiques du don dans la société française du XVI ${ }^{e}$ siècle : voir son Essai sur le don (Paris : Seuil, 2003). Dans la lignée de ces travaux, Claire Sicard détaille, dans la deuxième partie de sa thèse, les formes que peuvent prendre les " dons " : il peut s'agir d'un livre (ou d'un échantillon d'œuvres), il peut s'agir de donner du plaisir, de donner son affection, de se donner soi-même, de donner l'immortalité... : voir Poésie et rapports sociaux autour de la cour de France (1538-1560) (thèse de doctorat, Université Paris 7, 2013).

27. Brach, Les Amours d'Aymée, 272. 
- envoi par Brach de poèmes de deuil à Nesmond, sollicitant explicitement ou implicitement une pièce en retour pour alimenter le tombeau poétique qu'il veut composer pour Aymée ;

- réponse par Nesmond, sous la forme d'un sonnet, effectivement présent, en troisième position, dans le manuscrit du tombeau ;

- continuation de la composition du recueil et rédaction de la dédicace par Brach ;

- dédicace qui occasionne elle-même un remerciement de la part de Nesmond ${ }^{28}$.

Ce processus est sans terminus ad quem si ce n'est la date de la mort de Brach (1605) puisqu'il n'y a pas de publication imprimée qui en découle. Notons que la circulation des pièces poétiques met en relation le poète avec de nombreuses personnes : ainsi, les pièces 39,40 et 41 mettent en scène un dialogue avec Françoise de Terissan, qui écrivit une ode et deux sonnets pour figurer dans le tombeau d'Aymée.

De fait, le réseau que Pierre de Brach exhibe dans le diptyque «Larmes/ Tombeau $»^{29}$ n'est pas seulement un réseau sociologique, mais un réseau d'interactions de publication. En cela, la communauté de lecture formée est soudée non seulement par la reconnaissance de goûts partagés, mais aussi par des obligations éthiques d'attention et de réponse par poésie interposée, ce qui motive l'écriture et la lecture et leur impose une temporalité médiée par les temps de réponse, sur un support composite réunissant morceaux imprimés et manuscrits, dans un état inachevé-lisible. Le public que Pierre de Brach construit pour son recueil est en réalité un public restreint bien particulier, à la fois lecteur et co-auteur. Le poète constitue, pièce par pièce, son public. Ce processus de circulation participe de manière effective à l'augmentation et à l'orientation du recueil poétique.

28. Reinhold Dezeimeris pense avoir identifié cette pièce : voir Euvres poétiques de Pierre de Brach, vol. 1, 202, et Brach, Les Amours d'Aymée, 272.

29. Pour la liste des contributeurs, voir l'appendice III dans l'édition des Amours d'Aymée de Jasmine Dawkins (373-374) qui recense seulement les participants au Tombeau et la forme de leur contribution poétique, et pour les pièces mêmes, voir l'édition des Euvres poétiques de Pierre de Brach par Reinhold Dezeimeris. 
Christofle du Pré, au contraire, fait paraître très rapidement son recueil de Larmes funebres, publié en octobre de l'année même de la mort de sa femme Antoinette de Faucon, décédée en juillet.

Le deuxième sonnet du recueil présente un intéressant récit généalogique del'inspiration poétique, avec une rupture entre l'avant et l'après de l'événement de la mort de la femme aimée, rupture mise en valeur à la volte du sonnet pour exprimer la conversion du chant amoureux en chant endeuillé :

Le premier jour qu'Amour me vestit de souci,

Et me feit demander le loyer de ma peine,

Il m'apprist à chanter, et ma Muze hautaine

(Reconfort d'amoureux) ne chantoit que merci :

Fust que Phebus esteint vist son œil obscurci,

Ou fust qu'il flamboyast sur le sein de la plaine, Je chantoy sans cesser, et mon chant de Sereine Amolit dans un cœur un rocher endurci.

Ores dessous la Mort have, blesme et defaite, Qui veut voir le pourtrait d'une image muette, M’approche, il me verra sans poumons et sans vois :

Non, il m'orra chanter, mais ces chansons contraintes,

Publiant mon malheur, serviront cette fois

De clairon et de trompe à mes tristes complaintes. ${ }^{30}$

La « Muze hautaine » du vers 3 cède sa place de patronne à « la Mort have, blesme et defaite » au vers 9. Le poète exprime le « jaillissement» de sa plainte et fait de ses lecteurs les témoins de cette expression de la douleur : c'est là que la si célèbre amorce pétrarquienne "Qui voudra voir » est reprise, exhibant la filiation poétique en même temps que sa transfiguration par la nouveauté et par la réalité de l'inspiration de Christofle du Pré. L'emploi du participe présent "Publiant» au vers 13 est à noter tout particulièrement, puisqu'il s'agit ici de rendre public le deuil, de le faire connaitre à un public que l'on imagine 
le plus large possible au vu des instruments bruyants qui sont convoqués (clairon et trompe). L'intention de "large diffusion " professée par le sonnet (au sein de la "fiction " poétique, strictement parlant) est en accord avec le choix d'adresser le liminaire "Aux lecteurs » (adresse indéterminée) et avec la rapidité du processus de publication des Larmes funebres qui paraissent trois mois seulement après la mort de l'épouse du poète. Le processus de publication imprimé est ici investi d’une intention de diffusion large et immédiate.

Quelques restrictions sont toutefois apportées au sein du liminaire "Aux lecteurs " dans la définition du public visé. Christofle du Pré anticipe les moqueries qu'il encourt en choisissant de chanter son épouse, c'est-à-dire un être qui n’aurait pas a priori la " dignité littéraire » des grandes dames aux surnoms mythologiques chantées dans de nombreux canzonieri.

\section{Aux lecteurs}

Comme parmi la troupe des Astres on en voit de bons et de mauvais : et comme dans un grand pré il y a de diverses sortes d'herbes, les unes salutaires, les autres mortelles : aussi y a-il entre les humains des hommes de bonne nature, et qui l'ame saincte et nette, louent les choses honnestes et vertueuses : les autres de perverse et maligne, se moquants de toutes œuvres bonnes et louables, pour le moins qui d'un branslement de teste veulent donner à cognoistre ce que leur cœur n'oze du tout mettre en evidence. Voyla pourquoy je m'asseure qu'une infinité de Rogers-bontemps, plustost allechez du vice que de la vertu, lizans ces Sonnets riront dequoy je louë celle que le Ciel m'avoit destinee, et que je plore la perte que j'en ay faicte. Mais me souciant peu de telle rizee, j'aime mieux estre veu du Soleil en l'eclipse, que de la Lune en sa plus claire lumiere. Et si bien souvent l'homme louë le petit chien turquet de Madame, ou sa chambriere, et quelque bonne piece, l'honnorant du tiltre de femme d'honneur et de reputation : pourquoy ne le donneray-je à celle qui le merite, et qui a toujours vescu en ce degré ? Se moque donc qui voudra, et soyent encore en la France les vices plus desbordez qu'ils ne sont. ${ }^{31}$

La restriction du public appelé vise, sous la qualification de "Rogers-bontemps ", les poètes de cour ; Jamyn et Desportes, pour leurs railleries sur le 
mariage, sont probablement en ligne de mire. Du Pré fonde ainsi la définition de son public sur un critère moral. Cependant, même si le discours tenu restreint le public convié, le lectorat visé reste large. Cet apparent paradoxe se résout si l'on considère que la fonction de ce liminaire est moins d'exclure $a$ priori des lecteurs que de les convertir à l'éthique littéraire qui est défendue et de les amener à considérer le sujet du deuil conjugal comme digne d'être hautement traités2.

Pierre de Brach s'attend également à une incompréhension et explique longuement à son dédicataire Monsieur de Nesmond quel serait le public capable de comprendre et digne de recevoir les larmes qu'il verse sur la mort de son épouse. Le poète prend pour critère décisif la communauté d'expérience : pour comprendre et donc trouver une forme d'agrément aux vers qu'il publie, il faut avoir une expérience du mariage au moins en partie comparable à la sienne.

Monsieur, j'ay longuement esté en doubte à qui je donneroy ces vers d'un mary qui regrette et lamante la mort de sa femme ; car je craignois, si je les donnois à quelqu'un qui n'eust point encores esprouvé les douceurs d'un heureux et bien accordant mariage, que celuy là ne pourroit pas juger avecques justice si justemant je me plain de ma perte, la perte d'un bien ne se pouvant estimer que par la pocession et par la privation. Je craignoy, si je les donnois à quelqu'un à la noce duquel Hymen eust alumé son flambeau, que je les pourroy donner à tel qui, au lieu de me plaindre, se plaindroit soy mesme dequoy il n'estoit à mesme, et à qui mes larmes en mesme accidant seroient autant de ris et mes regrets autant de contentements. Et en fin je voioy qu'à tel que je les peusse donner, ce seroit toujours un ennuieux et facheus presant que des larmes, qui ne viennent jamais à nous qu'avecques la triste occasion ou d'une douleur qui nous presse ou d'une pitié qui nous esmeut ou d'une perte qui nous afflige; si bien que tout le monde les fuit et les hait. ${ }^{33}$

Le public « appelé » pour les Regrets et larmes funebres est défini, via la personne emblématique de Monsieur de Nesmond, sous les traits d'un marié aimant, 
capable de comprendre la peine que la perte de sa femme a causée à Pierre de Brach. Les deux poètes définissent ainsi quel doit être, qualitativement, le public capable d'apprécier leurs vers. Cependant, si le procédé est comparable sur ce choix qualitatif du public, une différence demeure dans la mesure où Christofle du Pré fait imprimer ses pièces poétiques, ce qui constitue une ouverture, tandis que Pierre de Brach maintient les siennes dans un circuit fermé.

\section{Donner sens aux temporalités de publication}

Les descriptions de ces quatre processus de publication permettent de remarquer la cohérence poétique entre les contenus des recueils et les temporalités de diffusion et de réception du livre. En effet, dans la mesure où le recueil peut garder trace d'événements et de circonstances de partage des textes, dans les cas qui nous occupent qui sont la célébration d'un amour royal et la déploration d'un deuil, le recueil prend une fonction mémorielle.

Chez Jamyn et chez Ronsard, il s'agit d'une mémoire historique : les vers pour Eurymedon et Callirée deviennent un "document " déchiffrable avec clé, attestant d'un événement courtisan qui reste en partie insoluble dans les sections pétrarquistes des œuvres des deux poètes.

Chez Du Pré, il s'agit d'un livre commémoratif. Il est celui qui donne le plus à son livre l'autonomie d'un monument, capable de garder le souvenir de la défunte et de sublimer ce souvenir. C'est ce qu'annonce la fin du liminaire «Aux lecteurs »: «Je veux estre aussi le premier qui face voler l'amitié qu'on doit porter à sa fidelle moitié, essayant d'éternizer la mienne pour le contrepoix de son amour envers le mien $»^{34}$. La fonction «éternisatrice» du recueil est explicitement affirmée.

Enfin, le cas de Brach est particulier en raison de l'absence de publication imprimée et de la composition même du manuscrit. La fonction mémorielle semble être de l'ordre de l'exercice du deuil. Il s'agit en effet de faire durer le deuil, en acte, en continuant à écrire et à faire circuler ses poèmes au sein d'un cercle compréhensif, dans une attitude de rétention par rapport à la publication imprimée. Or, la préoccupation de la durée du deuil est un thème récurrent dans les sonnets de Pierre de Brach. Ainsi le sonnet 5 qui prend pour sujet l'habit de deuil : 
Triste habit, je vous pren, non pas pour une année ;

D'une amour qui ne peut du temps être bornée

Le deuil dedans un an ne se peut arrester. ${ }^{35}$

L'idée que le deuil dure par les vers mêmes est aussi le sujet du dialogue avec Françoise de Terissan dans les pièces 39 à 41 évoquées plus haut :

Pour moy rien en terre il ne reste,

Mon bien terrestre faict celeste

Rend tout celeste mon pencer ;

Le deuil ses tablettes me laisse;

L'amour de la mort qui me blesse

Mille vers m'y faict retrasser. ${ }^{36}$

Car d'aymer un corps mort je ne puis me distraire ${ }^{37}$.

Enfin, le sonnet 53 contient encore un reproche à ses amis qui l'enjoignent à changer de sujet pour quitter sa tristesse :

Que vous estes fascheux, mes amis, de me dire

Que je laisse meshuy ce funeste argumant ;

Voulez-vous qu'en mon deuil on voie lachemant

Mon vouloir retirer où mon devoir me tire?

Vous ne verrez ma main d'autre sujet escrire

Ny jetter mes dessains sur autre fondemant

Que sur la mort d'Aymee, haussant un monumant

Qui sera dans mes vers marque de mon martire. ${ }^{38}$

Ce sonnet 53 affiche le projet de monument commémoratif qui nous semble réalisé en tant que tel chez Du Pré ; néanmoins, quoi qu'en dise le poète, le circuit de publication qu'il choisit aboutit moins à « hausser un monument »

35. Brach, Les Amours d'Aymée, III, 5, v. 9-11, 278.

36. Brach, Les Amours d'Aymée, III, 39, v. 13-18, 323.

37. Brach, Les Amours d'Aymée, III, 40, v. 11, 324.

38. Brach, Les Amours d'Aymée, III, 53, v. 1-9, 336. 
qu'à conserver une mémoire en activité, survivant dans un certain cercle de relations sociales et dans un acte d'écriture continué. Le projet du poète semble avoir été de publier effectivement son manuscrit, si l'on en croit une note manuscrite relevée par l'abbé Goujet dans son exemplaire de la Bibliothèque de La Croix du Maine, au sujet d'une personne qui écrit s'être entretenue avec Pierre de Brach de son projet de laisser ses « Regrets funebres " paraître après sa mort ${ }^{39}$. Mais de fait, de son vivant, le processus de publication par lequel il fait lire ses poèmes implique de les lire dans un cadre herméneutique qui est moins celui du monument érigé pour le souvenir de la postérité que celui d'un entretien du souvenir qui fait durer le deuil. L'échange des pièces poétiques semble participer de la consolation, ce qui entre en tension contradictoire avec les poèmes de plainte endeuillée qui construisent un ethos de poète inconsolable.

Comment les deux sections d'œuvres de Ronsard et Jamyn sur les amours d'Eurymédon et Callirée et les deux recueils « funèbres » de Brach et Du Pré s'inscrivent-ils dans des temporalités historiques par les processus mêmes de leur divulgation à certains publics ? En ce qui concerne Ronsard et Jamyn, le public restreint auquel les vers s'adressent (le roi et sa maîtresse, la cour) contribue lui-même à créer un premier cadre herméneutique pour la pleine intelligibilité de ces vers. Ce cadre herméneutique est ensuite doublement déplacé dans le recueil imprimé : par l'inscription dans une série intertexuelle d'autres vers amoureux qui relèvent des amours pétrarquistes, mais aussi par la possibilité de la lecture «à clé » dans laquelle les poèmes peuvent être appréciés pour leur façon de crypter l'allusion aux amours royales. La mise en recueil résout ainsi partiellement la péremption de l'événement. Chez Pierre de Brach, le public visé par la circulation des pièces poétiques qui constituent la section de « regrets et larmes funèbres » après la mort d'Aymée reste un public restreint et choisi tant que la publication imprimée est différée et retenue. À l'inverse enfin, chez Christofle du Pré, le public convoqué est un vaste lectorat, exception faite des moqueurs présumés (dont l'exclusion tient sans doute en bonne partie du jeu de positionnement dans le champ littéraire), et une visée « éternisatrice » est donnée à la publication du livre.

Quelles sont donc les conséquences de ces modalités d'implication des publics dans la publication sur la façon de comprendre la résonance de ces recueils avec le cadre générique pétrarquiste ? En prenant l'exemple de la 
dialectique verticale qui s'instaure entre les positions symboliques de la dame aimée et de l'amant, examinons comment l'existence de plusieurs publics destinataires dans le processus de publication implique certaines conditions de lisibilité concurrentes, vis-à-vis du seul cadre herméneutique du maniérisme pétrarquiste.

\section{Détournements poétiques et construction de valeurs}

L'hypothèse est que la signification de l'adaptation d'un motif poétique peut se trouver fondée sur des valeurs que seul le premier contexte de divulgation et de circulation rend pleinement intelligibles.

La fiction amoureuse telle qu'elle se présente dans le pétrarquisme (en particulier dans ses veines néo-platoniciennes) met couramment en scène la dame comme inaccessible dans sa perfection élevée, voire " céleste », par contraste avec la misère de l'amant, en position d'infériorité. Chez Ronsard et Jamyn d'une part et chez Du Pré et de Brach d'autre part, le détournement de cette dialectique des « positions » est un aspect qui cristallise le jeu sur la limite du code pétrarquiste.

\section{Inversions anti-pétrarquistes}

Chez Ronsard et Jamyn, l'application au roi de la rhétorique amoureuse joue sur un renversement des positions de pouvoir entre l'amant et sa dame. La puissance royale est contraire à la représentation courante du service amoureux : en effet, le roi étant forcément au-dessus de l'ensemble de ses sujets, la dame ne peut pas «l'élever » de la même façon qu'elle élèverait un amant ordinaire. La relation décrite entre le roi et sa maîtresse est donc adaptée aux statuts sociaux des protagonistes, et cette relation adaptée fait apparaitre, peut-être avec une forme d'ironie, une certaine vérité des rapports entre hommes et femmes à la cour, démasquant le jeu de dupes du " service » de l'amant envers sa « dame ».

Ainsi, dans les poèmes qu'Amadis Jamyn prête au roi (la plupart sont écrits à la première personne, la voix étant, fictivement, celle du roi, hormis une chanson fondée sur un récit à la troisième personne, mais qui comprend des paroles fictives du roi, rapportées au discours direct), l'accent est mis sur la grandeur royale hors du commun. Le roi doit « abaisser » sa grandeur ${ }^{40}$ pour

40. Jamyn, III, «Chanson », v. 27, 112. 
se mettre au niveau de sa dame, et le motif de l'élévation amoureuse aboutit à un traitement paradoxal. En effet, il est d'abord repris de manière tout à fait commune :
Ainsi l'Amour au Ciel m'attire l'ame, M'evaporant par sa gentille flame Tout l'imparfaict du terrestre fardeau :
Il fait mon corps léger comme un oyseau, Et de la terre il dérobe ma plante, Me soulevant de son æle volante : Je vole en l'air transporté de plaisir Pour toy mon cœur, mon sang, et mon desir. ${ }^{41}$

Mais ensuite, à la faveur d'une évocation de la danse, la légèreté hyperbolique $\mathrm{du}$ roi le rend inaccessible, ce qui crée une situation inverse à la situation habituelle, où c'est la dame qui l'est :

Donc en dansant j'ai le corps si leger

Que loin de terre au ciel j'irois loger,

Aigle d'amour, tant ma force amoureuse

N'est point vulgaire, ains haute et genereuse,

Si ta beauté me suivoit parmi l'air :

Mais tout soudain mon vol se vient caler,

Et retombant mes ailes je reserre,

Voyant ton pié demourer sur la terre,

Qui toutefois, leger, ne cede en rien

A ce que dit le vers Virgilien

De la guerriere et superbe Camille

Qui sur les bleds avec sa plante agille

Eust peu courir sans les épics toucher,

Et sur la mer sans l'attaindre marcher.

Las! que ferois-je en cette maison haute

De Jupiter, si de toy j'avais faute?

Et quel plaisir aurois-je entre les dieux

41. Jamyn, III, «Elegie », v. 31-38, 122. 
Sans le beau jour des astres de tes yeux?

$\mathrm{Ha}$ ! Tant s'en faut que monter je voulusse

$\mathrm{Au}$ Ciel sans toy, bien que voler j'y peusse :

Que si j'estois l'eternel commandeur,

Je quitterois l'Olympe et ma grandeur

Et descendrois sur la terre nourrice

Pour dedier mes ans à ton service. ${ }^{42}$

La concession accordée à la dame par la comparaison avec la Camille de l'Énéide ${ }^{43}$ ne fait rien à l'affaire : le roi est bel et bien d'un statut supérieur aux autres mortels, elle comprise. La propension du roi à s'envoler peut faire écho, dans une dégradation burlesque, à la mission royale qui est de recevoir sa puissance de Dieu et de rayonner de cette puissance.

Dans les «Stances» des « Amours d'Eurymedon et Callirée » de Ronsard, on observe une variation sur la même matière. Le roi y est " Demon ", être surnaturel intermédiaire entre humain et divin. Ronsard se montre plus antipétrarquiste encore, puisque le poète met en scène une récrimination contre la dame, accusée de ne pas aimer assez son amant pour le suivre dans les airs :

Je ressemble au Demon, qui ne se veut charger

D'un corps, ou s'il a corps, ce n'est qu'un air leger,

Pareil à ces vapeurs subtiles et menues,

Que le Soleil desseiche aux chauds jours de l'Esté.

Le mien du seul penser promptement emporté,

Distillé par l'Amour, se perd dedans les nues.

$[\ldots]$

La matiere de l'homme est pesante, et ne peut

Suivre l'esprit en haut, lors que l'esprit le veut,

Si Amour, la purgeant de sa flame estrangere,

N'affine son mortel. Voilà, Dame, pourquoy

Je cognois par raison que n'aimez tant que moy:

Si vous aimiez autant, vous seriez plus legere.

Entre les Dieux au ciel mon corps s'iroit assoir,

Si vous suiviez mon vol, quand nous ballons au soir

42. Jamyn, III, « Elegie », v. 61-84, 123.

43. Virgile, L'Énéide, VII, v. 808-811 (Paris : Les Belles Lettres, 1952), 41. 
Flanc à flanc, main à main, imitant l'Androgyne :

Tous deux dansans la Volte, ainsi que les Jumeaux,

Prendrions place au sejour des Astres les plus beaux,

Et serions dicts d'Amour à jamais le beau signe.

Où par faute d'aimer vous demeurez à bas,

La terre maugré moy vous attache les pas.

Vous estes paresseuse et au ciel je m'en-vole.

Mais à moitié chemin je m’arreste, et ne veux

Passer outre sans vous : sans y voler tous deux

Je ne voudrois me faire un citoyen du Pole. ${ }^{44}$

$\mathrm{Au}$ rebours des habitudes pétrarquistes, les rôles sont donc inversés : la dame n'est pas inaccessible, c'est le roi qui l'est. À quoi jouent ici les deux poètes? Tout d'abord à un plaisant jeu de connivence avec le roi, à travers une insistance sur sa générosité et une complicité masculine dans la raillerie d'un code lyrique qui idéalise à l'excès l'évertuement amoureux de l'amant et la position surélevée de la dame. Loin de souscrire à la tendance néo-platonicienne qui fait de la dame une médiatrice vers la beauté céleste, ces poèmes donnent au roi seul le pouvoir d'élever dans son sillage les hommes vers les cieux divins - les hommes, notons-le, mais pas la trop « paresseuse » maîtresse. La femme, dans le poème de Ronsard en particulier, est engluée dans la terre qui la retient ("La terre maugré moy vous attache les pas »), ce qui correspond au statut ontologique de la femme dans l'imaginaire aristotélicien, où elle n'est que matière réceptrice, sans force productrice ni spirituelle ${ }^{45}$. Or, dans le contexte de la cour, cette subversion du pan néo-platonicien du pétrarquisme favorable à la reconnaissance des facultés intellectuelles et morales féminines dénie à la maitresse du roi une réelle position d'influence.

\section{L'inaccessibilité réelle}

Dans les recueils de deuil, le détournement est bien sûr tout différent. Le statut inaccessible de la dame et l'effort d'élévation de l'amant-poète sont réinvestis dans la mesure où la femme aimée est réellement (si l'on peut dire) « au ciel ».

44. Ronsard, «Stances », v. 49-54 et v. 61-78, 146-147.

45. Voir Aristote, De la génération des animaux, I, 2, 716a (Paris : Les Belles Lettres, 1961), 3-4. 
L'excès hyperbolique de la plainte, si couramment reproché aux poètes pétrarquistes ${ }^{46}$, est soudain fondé, dans la fiction même, par le recours au « dehors » de la fiction. Du Pré appelle ses lecteurs à apprécier cette vérité par contraste avec une poésie de cour futile. Pierre de Brach insiste particulièrement sur la vérité de la parole, comme le montre Olivier Pot ${ }^{47}$, en allant jusqu'à se condamner lui-même d'avoir attribué à sa femme le nom d'« Aymée " "pour chanter d'un nom fainct un amour véritable ${ }^{48}$. Un regain de vigueur est donné à la fictionnalisation des événements biographiques. Le topos poétique, pour usé qu'il soit par les pétrarquistes français, fait l'objet d'un renouvellement par la circonstance, et toute la subtilité de ce renouvellement est de renouer avec l'événement du deuil présent originellement dans l'œuvre pétrarquienne.

La dialectique qui s'instaure chez Christofle du Pré entre la dame « au ciel » et le poète resté à terre est analysée par André Gendre ${ }^{49}$, qui éclaire la façon dont la forme sonnet est exploitée, dans sa structure logique et rythmique, pour mettre en scène l'écart entre le ciel et le poète, et la tentative de transformer cet écart douloureux en élan vertueux. Le sonnet 53 est exemplaire de l'affirmation d'une volonté d'élévation :

\section{[...] Ce depart tant estrange}

Pourroit-il me geler et me faire moins chaud?

Mettra-t-il mon amour sous la tombe couverte?

Non non, ains pour tirer profit de ma perte,

Despouillant le manteau des abus de la chair,

Sur mes pensers aislez d'une plume bien forte,

Et guidé du respect que mon ame vous porte,

Je veux sonder le ciel et vous y rechercher. ${ }^{50}$

46. Voir Jean-Yves Vialleton, « Le Pétrarque des antipétrarquistes français des années 1550 », Cahiers d'études italiennes 4 (2006), 99-115.

47. Olivier Pot, «Le cercle des poètes disparus : Pierre de Brach et l'école de la mélancolie », Albineana, Cahiers d'Aubigné 22 (2010), 179-199.

48. Brach, Les Amours d'Aymée, III, 51, v. 4, 335.

49. André Gendre, «Sonnets et odes de Christofle Du Pré : nouveauté au seuil du baroque », Albineana, Cahiers d'Aubigné 22 (2010), 329-345.

50. Du Pré, Les Larmes funebres, 53, v. 7-14, 205. 
L'intention de "tirer profit de [l]a perte " revient à faire de l'épreuve de la souffrance le levier d'une plus grande force d'âme : il s'agit en quelque sorte d'une instrumentalisation vertueuse ; mais la chute du sonnet est une sorte de rechute idolâtre, où la quête spirituelle « dépouillée » de l'attrait pour les " abus de la chair » aboutit certes à lever les yeux vers le ciel, mais pour y chercher la défunte, et non pas Dieu. Le deuil a chez Du Pré un caractère indépassable, même lorsque le poète utilise une dialectique chrétienne et néoplatonicienne du dépassement des attachements terrestres. Suivant le même procédé, le dernier sonnet semble apporter un apaisement, mais le dernier vers, dans une ultime hyperbate dont la force de « contrepoint » est relevée par Pierre Martin $^{51}$, laisse en quelque sorte expirer la voix du poète vers le ciel : « toutesfois je confesse / Qu'en mon infirmité je soupire vers vous ${ }^{52}$.

D’un point de vue formel, le recueil de Du Pré est celui qui joue le plus de l'intégration dans l'intertextualité des canzonieri, et il est intéressant de signaler qu'il exploite des réminiscences scéviennes ${ }^{53}$. En effet, Du Pré reprend et réécrit des vers de Delie, object de plus haulte vertu de Maurice Scève, premier recueil pétrarquiste publié en France en 1544. Ce faisant, le poète lance un double signal : d'une part, il réactive une filiation poétique passée de mode dans la décennie 1570 où il écrit, optant pour une forme d'inactualité (en écho avec l'exclusion du public des poètes de cour dans son liminaire « Aux lecteurs »); d'autre part, il met ainsi ses poèmes en écho avec ceux d'un recueil dont l'aboutissement est une éternité acquise pour deux, pour le poète et sa dame : « Nostre Genevre ainsi doncques vivra / Non offensé d'aulcun mortel Letharge $\aleph^{54}$. Le point final des Larmes funebres reste plus sombre, mais c'est en reprenant le liminaire "Aux lecteurs », où s'affirme le projet d'« éternizer » l'épouse défunte, que l'on peut comprendre que la fonction commémorative n'est pas une affaire littéraire, mais une affaire de publication. Autrement dit, les mots n'ont pas à transcender l'expérience du malheur, c'est la publication du livre qui réalise cette transcendance.

51. Du Pré, Les Larmes funebres, 141.

52. Du Pré, Les Larmes funebres, 75, v. 13-14, 227.

53. Ces reprises sont repérées et recensées par Pierre Martin dans son édition des Larmes funebres de Christofle du Pré.

54. Maurice Scève, Delie, object de plus haulte vertu (Paris : Société des Textes Français Moderne, 2001), 449 , v. 9-10, 305. 
En regard de la poésie de Christofle du Pré, celle de Pierre de Brach contraste par sa dimension concrète. La vie endeuillée est décrite comme une errance sur la terre, se déploie dans l'horizontalité. De nombreux sonnets sont des « visites » aux lieux anciennement partagés (la maison, l'église, un chemin, un jardin...). Ainsi au sonnet 24 :

Sombre allée, en lauriers espaissement ombreuse,

Qui me sert de carriere, où je vais si souvant,

Esperonné du deuil, cerchant et ne trouvant

Ce que m’a desrobé la tombe tenebreuse,

Ta belle promenade un temps me fust heureuse

Quand nos devis alloient tes feuilles esmouvant;

Mais, ores que mes pleurs vont ta terre abrevant,

Autant que tu m'as pleu je te trouve ennuieuse..$^{55}$

Ce poème est au cœur de la séquence des sonnets 23,24 et 25 qui prennent pour cadre la campagne environnante. Le poète est ici en interaction avec la terre, dans laquelle est creusée la tombe et qui s'imbibe des larmes versées. Il se figure marchant seul, dans le souvenir de la promenade à deux, symbole de l'union conjugale où les deux personnes vont côte à côte, au même niveau, par opposition à une relation où l'une doit s'élever vers l'autre, modèle de la relation pétrarquiste entre l'amant et sa dame.

La description de l'« ici-bas " suit le cours du temps terrestre et met en scène la succession des saisons. Les sonnets 33 et 34 voient ainsi l'arrivée de l'hiver qui conforte l'humeur du poète, et le sonnet 34 est un tableau des «ombres», tant corporelles que spirituelles, errantes en cette saison morte:

Ombre (car je ne suis qu'une ombre seulemant

D'un corps perdant un corps qui a perdu son estre),

L'ombre je vais cerchant où l'ombre souloit estre

Des arbres despouillez de leur verd ornement.

Ombre, je hay mon ombre, en ce qu'au mouvemant

Une marque de vie elle faict apparoistre. ${ }^{56}$

55. Brach, Les Amours d'Aymée, III, 24, v. 1-8, 306.

56. Brach, Les Amours d'Aymée, III, 34, v. 1-6, 316. 
Ce sonnet, dont les premiers vers sont fondés sur un jeu de démultiplication des « ombres » à la faveur d'une diaphore, est marqué par le désir de mort qui est un thème récurrent dans le recueil. S'il s'agit bien de mourir pour rejoindre l'épouse défunte, le poète développe toutefois une poétique fondée davantage sur la description pathétique d'une vie désertée que sur l'exaltation de l'ascension céleste.

\section{Divergences de valeurs}

Ce qu'il importe de souligner est que la reprise du code pétrarquiste est de nature critique. Chez Ronsard et Jamyn, cette reprise est le moyen d'ironiser sur les relations amoureuses courtisanes. Chez Du Pré et Brach, l'inscription dans le paradigme pétrarquiste met en exergue les dimensions pathétique et éthique de leur poésie, dans la mesure où la réalité du décès rend de facto crédibles l'ethos auctorial et l'épreuve de l'absence de la femme aimée.

Dans les quatre cas, ces « avatars » de canzonieri relèvent de deux formes d'antipétrarquisme : chez Ronsard et Jamyn, c'est un antipétrarquisme fondé sur la dévaluation du discours pétrarquiste, grâce au jeu d'inversion parodique; chez Brach et Du Pré, c'est un antipétrarquisme s'exerçant contre les pétrarquistes de cour, qui vise au contraire à redonner du crédit à l'accident biographique de la perte de l'être aimé. Ainsi, deux types de rapports à la fiction pétrarquiste se dégagent. Dans les poèmes sur les amours d'Eurymedon et de Callirée, l'artifice de la fiction est assumé jusque dans le maniérisme le plus invraisemblable. Il s'agit de renverser les positions topiques de la dame et de l'amant pétrarquiste, et ce renversement a du sens, premièrement à destination du public de la cour habitué à ces images topiques, et deuxièmement en résonance avec les recueils d'amour dans la série desquels ces vers pour Eurymedon et Callirée se trouvent publiés en recueils pour un public large. Dans les recueils funèbres, au contraire, le détournement critique du recueil amoureux se fait par une posture de dénonciation de la fiction, au profit d'une vérité du deuil.

\section{Conclusion}

Nous avons cherché à rendre compte des traces des premiers processus par lesquels des vers amoureux de cour et des vers amoureux de deuil sont livrés à un premier public afin de comprendre la portée de ces processus sur le sens 
des recueils. Le format générique du canzoniere imprimé neutralise-t-il le sens déterminé par la primauté de l'adresse à un public restreint ? À retracer les processus de publication, il semble bien qu'un effet de neutralisation se produise, dans la mesure où la publication imprimée " éloigne » les circonstances et les destinataires premiers; mais les interactions entre les poètes et leurs publics de circonstance restent porteuses de signification alors même que les poèmes invitent à une herméneutique liée à leur classement générique. Aborder ces poèmes comme "poèmes de genre ", en esthète qui goûterait les audaces maniéristes comme pur jeu, ne constitue donc pas une herméneutique complète, tandis que la prise en compte des premières destinations fonde une herméneutique adéquate. En effet, c'est en écrivant pour le souverain, par lui et en son nom que Ronsard et Jamyn renversent les positions et les rôles de l'amant et de la dame. Quant à Brach et à Du Pré, ils refondent une poétique de la perte de l'être aimé, Du Pré en érigeant le recueil en monument de crise, et Brach en faisant durer le deuil, en transcrivant un continuel retardement de la consolation.

Ce qui apparaît à la comparaison de Ronsard et Jamyn, d'une part, et de Du Pré et Brach, d'autre part, c'est une scission des valeurs à la fois poétiques et éthiques. Certes, dans tous les cas, les poètes font de l'imitation critique, s'appropriant un code poétique à travers un réglage de ce qu'ils rejettent ou reprennent ; mais le dissensus apparaît, entre ces deux couples de poètes, dans l'élection du matériau à transformer, et cela de manière étroitement liée à leur public cible. C'est sur le choix de ce qui est identifié comme discours porteur de valeur dans le pétrarquisme qu'ils se distinguent. Pour agrémenter les amours royales, Ronsard et Jamyn ouvrent une brèche dans la topique pétrarquiste établissant les rapports d'influence et de domination entre amant et dame. En revanche, pour développer une poésie du deuil, Brach et Du Pré retiennent tout le langage pétrarquiste de l'épreuve psychologique et spirituelle, et l'appliquent à un amour conjugal, ce qui constitue une reprise tout aussi subversive. 